Aleksander Gemel

BARTOSZ ŻUKOWSKI

\title{
SEMIOTICS, SIGNALING GAMES AND MEANING
}

\section{Introduction}

The 20th-century discussion on meaning was dominated by two distinct schools of thought - by that of naturalistic semantics and by that of structural semiotics. Although the two have traditionally been viewed as conflicting approaches, the main aim of this paper is to show that they can be seen as complementary to one another. In order to achieve this objective, we will examine the advantages and drawbacks of both approaches (represented, respectively, by Eco's semiotics and Skyrms' game-theoretical semantics). The results obtained this way provide a basis for further development of hybrid system by combining features of structural and naturalistic models.

\section{Structural semiotics. Between signalization and signification}

The distinctive feature of structural semiotics is a strict separation of two independent semiotic systems: signalization and signification. At the same time, in accordance with the basic premise of structural semiotics, it is only through both of these autonomic systems that a proper communication process can work successfully. Considered in itself, signalization is simply "the passage of a signal (not necessarily a sign) from a source (through a transmitter, along a channel) to a destination" (Eco, 1976: 8). The signals used for this process derive from a special kind of semiotic system called s-code, resulting from the division of some natural or artificial continuum. An essential feature 
of s-code is that all of the primary system components are isolated arbitrarily (ibid.: 77). Nevertheless, when selected, they are to form a well-organized, independent system, in which the identity of each unit is completely determined by reference to others, and hence specified by its position in the whole (ibid.: 38). The system established in this way is an internally structured matrix with certain combinatorial properties (determined by a set of combinatorial rules). As a result, it possesses also some informational potential. It is precisely (mathematically) defined by the intrinsic properties of the system (arrangement of elements and combinatorial rules), determining the amount of information possible to transmit. So configured, the system can operate autonomously - without any semantic reference: "s-codes are systems or 'structures' that can also subsist independently of any sort of significant or communicative purpose" (ibid.). Consequently, a purely signaling system can be considered as a communication system only in a purely mathematical sense - as a combinatorial syntactic structure, able to convey a certain amount of information, but devoid of any meaning in itself: "In a machine-to-machine process the signal has no power to signify insofar as it may determine the destination sub specie stimuli. In this case we have no signification, but we have the passage of some information" (ibid.: 8); "A signal is a pertinent unit of a system that may be an expression system ordered to a content, but could also be a physical system without any semiotic purpose; as such it is studied by information theory in the stricter sense of the term. A signal can be a stimulus that does not mean anything but causes or elicits something" (ibid.: 48). In effect, Eco's signalization is perfectly consistent with the transmission communication model proposed by Shannon and Weaver (1949) as a starting point for the mathematical theory of communication (Eco, 1976: 42-44).

A signaling system serves as a basis for a signifying system. Notwithstanding this, the assumption underlying the whole semiotic theory of language is that the two systems remain functionally independent of each other. ${ }^{1}$ This central idea of structural semiotics seems to be an echo of the separation between semantics and syntax in logic and formal linguistics of the first half of the 20th century. The essence of signification is to establish a correlation of some units of a given s-code, considered as an expression system (plane), with the units of some other

1 "A signification system is an autonomous semiotic construct that has an abstract mode of existence independent of any possible communicative act it makes possible” (Eco, 1976: 9).

\section{8 \& COgNItIVE SCIENCE}


s-code treated as a content system (plan) (ibid.: 50). The fundamental difference between signalization and signification is therefore that the latter involves an interpretative (decoding) response of the receiver, which is made possible by the previously established convention of correlating signals with contents: "When the destination [of a communicative process] is a human being, or 'addressee' $[\ldots]$ we are on the contrary witnessing a process of signification - provided that the signal is not merely a stimulus but arouses an interpretative response in the addressee. This process is made possible by the existence of a code" (ibid.: 8). By contrast with signalization, signification is a process within which signals refer to certain units of content and, thus, become units of meaning, which situates signification as a transmission of signs.

What is most important, however, is that both systems (plans) of expression and of content are structures, or systems of s-code type. What is being correlated here are, therefore, two independent and arbitrarily construed systems - two autonomous combinatorial matrices, organized internally as systems of positions and oppositions. And it is precisely the correlation between particular elements of such s-codes which is called "a sign-function" or simply "a sign". A sign (sign-function) is, therefore, a relationship linking two different s-codes, one acting as an expression plan and the other being a content plan. Furthermore, the method used to correlate both (i.e. to assign some content to a signal) is based on convention only. In other words, the set of correlating rules, called "a code", is purely conventional. It goes simply as follows: "When a code apportions the elements of conveying system to the elements of a conveyed system, the former becomes the expression of the latter and the latter becomes the content of the former" (ibid.: 48). All the observed complexity of structural semantics results from repeating and accumulating such correlations. For example, a particular sign-function (i.e. a particular relationship between an element of a system of expression and an element of a system of content), called denotation, can be easily correlated with an element of a third system (s-code), thereby forming a higher-order sign-function, called "connotation" (ibid.: 54-57). The resulting connotative code consists of two functives: a pre-existing sign function and some element of a third s-code. Repeated many times, this process gives rise to the formation of a desirable rich semiotic system. All this clearly proves the dependence of the theory of semiotics on structural linguistics.

There are, no doubt, some significant advantages of this approach. The first and foremost of them is substantial flexibility of the structural semiotics. 
It provides a fertile ground for generation of new meanings and transformation of the current semantic field. At the same time, it seems to adequately reflect the dynamics of natural language, prone to constant reconfiguration of meanings. Thus, structural semiotics can rightly claim to be the 'logic of culture' (ibid.: 3). The aforementioned flexibility results from a combination of several features indicated above. Let us summarize them briefly as follows:

(a) Functional independence of signalization and signification - both systems are considered as autonomous combinatorial structures (s-codes).

(b) Arbitrary construction of s-codes of all types and levels. This feature is of special importance for the design of the plan of expression (ibid.: 77).

(c) Conventionality of the rules of linking the elements of the plan of expression with the elements of the plan of content.

(d) Rejection of the so-called 'extensional fallacy' (ibid.: 62-66). One of the most important features of structural semiotics not mentioned so far is rejection of referential semantics, correlating signs with some extra-semiotic objects: "From a semiotic point of view" the meaning of a term "can only be a cultural unit", understood as an element of some arbitrary construed matrix (ibid.: 67).

As a result of these assumptions, the semiotic system achieves the desired flexibility. It is easily susceptible to transformation - new units of content can be generated from within the system, by transformation of the existing semiotic infrastructure. In other words, the system has the capacity to freely evolve, just like culture itself.

Despite the abovementioned advantages of this system, it does still suffer from certain weaknesses, which should be overcome by partial naturalization. The first weakness has to do with the content plan - the problem is the absolute arbitrariness of the starting units. In fact, cognitive, anthropological and psychological studies revealed that there are some content universals common to all human cultures, which argues for the existence of natural determinants of content system (Bickerton, 2009; Bickerton, Szathmary, 2009; Brinck, Gärdenfors, 2003; Gärdenfors, 2004; Deacon, 1997; Ollera, Griebel, 2004). The second weakness is the avoidance of the question of the original sign correlations. Although Eco claims that the explanation of this issue requires a reference to the natural, pre-cultural conditions (Eco, 1976: 58-59, 77), he refrains from discussing this matter, probably because of the fear of falling into extensional fallacy (which, in our opinion, is excessive). Finally, the third problem with structural semiotics is its panlinguisticism. In order to fully reflect the actual functioning of

\section{0 \&) COGNITIVE SCIENCE}


language, structural semiotics must be supplemented with at least rudimentary external reference (correspondence to an extra-linguistic reality).

In conclusion, structural semiotics should be enriched with some components of naturalistic semantics, without, however, depriving it of its flexibility. Semiotics, for its part, can provide naturalistic models with generative/transformative potential, making them more flexible. A new hybrid system developed this way will feature the best advantages of both approaches. In the next section, we present an example of typically naturalistic semantics, which might serve as a starting point for such synthesis.

\section{Signaling games}

To illustrate the naturalistic approach to the question of coding conventions emergence and the fixation of meaning, we will use signaling games model, originally proposed by D.K. Lewis and developed by B. Skyrms (Lewis, 2002; Skyrms, 2010). This model provides a game theoretical instrument, which helps us to explain the genesis of such semiotic phenomena as code and meaning. Thanks to the game-theoretic framework, the concept of communication process in signaling games is recognized as a game between the sender and the receiver. The intention of Lewis' game is to provide a model of language and its semantic content genesis within a community devoid of any language system. Moreover, it is important to emphasize the fact, widely pointed out by Lewis, that a language constituted as a product of a sender-receiver game can only take a form of a fairly primitive, rudimentary proto-language (Lewis, 2002: 160). The mature form of a language system can potentially evolve from its proto-form, but Lewis does not deal with this issue. The mechanism of the mature language system formation is also not the subject of this paper, if only due to the degree of its complexity.

Lewis defines signaling game as a type of situation involving at least two agents (one in the role of a sender and the other as a receiver ${ }^{2}$ ). It meets four basic conditions. According to the first condition, at least one of several states of affairs occurs. States $\left(S_{1}, \ldots, S_{n}\right)$ are randomly picked by nature. In fact, random selection means that the occurrence of a particular state can be attributed with a certain probability. What is also important, in contrast to the sender, the receiver

\footnotetext{
${ }^{2}$ Lewis calls them communicator and audience (Lewis, 2002: 130-132).
} 
occupies a privileged position to observe and correctly identify a given state of reality. According to the second rule of the game, the sender, having observed one of the states $\left(S_{1}, \ldots, S_{m}\right)$, takes one of several alternative measures $\left(\sigma_{1}, \ldots\right.$, $\left.\sigma_{n}\right)$ called signals. The set of signals must be greater or at least equal to the set of states $(\mathrm{n} \geq \mathrm{m})$. Taking action $\sigma_{i}$ is equivalent to sending the signal to the receiver. The ontological nature of the signal is not pre-defined (it can be a sound, gesture, smell, etc.), since the signal is considered only in functional terms, i.e., its essence is to evoke a specific reaction of the receiver. Moreover, sending the signal by the sender does not have to be intentional or even conscious. Similarly to the first condition, the receiver is in a good position to receive the sent signal. The channel is not noisy, although adding noise to the model is possible by manipulating the prior probability of the signal. According to the third rule, the receiver, after observing and identifying the signal, takes one of several alternative reactions $\left(R_{1}, \ldots, R_{n}\right)$. Importantly, the receiver takes action based only on the received signal without knowing anything about the state of the reality. The fourth signaling game condition is that both players have a set of strategies (contingency plans). The strategy of the sender consists in sending a specific signal according to a given state of affairs. In mathematical terms, this corresponds to function $F_{s}$ associating one of the states in a set of states $\left\{S_{i}\right\}$ with a specific signal in a set of signals $\left\{\sigma_{k}\right\}$. On the other hand, you can specify the receiver's strategy as a function assigning the signal to the reaction. It is mathematically expressed as function $F_{r}$ assigning function $\left\{\sigma_{k}\right\}$ to $\left\{R_{j}\right\}$. Lewis describes the combination of the sender's and receiver's strategies $\left(F_{s}\right.$ and $\left.F_{r}\right)$ which provides the relationship between the reaction of the receiver and the state of the affairs $\left\langle F_{n}, F_{r}\right\rangle$ as the signaling system (Lewis, 2002: 130-132).

The essence of fixing a particular signaling convention is, therefore, the correlation between the reaction and the existing state of affairs, based on a proper signal. This correlation is effected through positive payoffs in the game. If the reaction of the receiver given a specific state of $S_{a}$ leads to positive payoffs for both players, then signaling convention gets fixed. In general, reaction is considered to be proper and signal is said to be adequate if the receiver, after getting the signal, takes an action that he would take in the case of a direct experience of the state of affairs.

This model can be presented in a simplified version of two states, two signals, and two reactions in the following graphic form:

\section{2 \&, COGNITIVE SCIENCE}




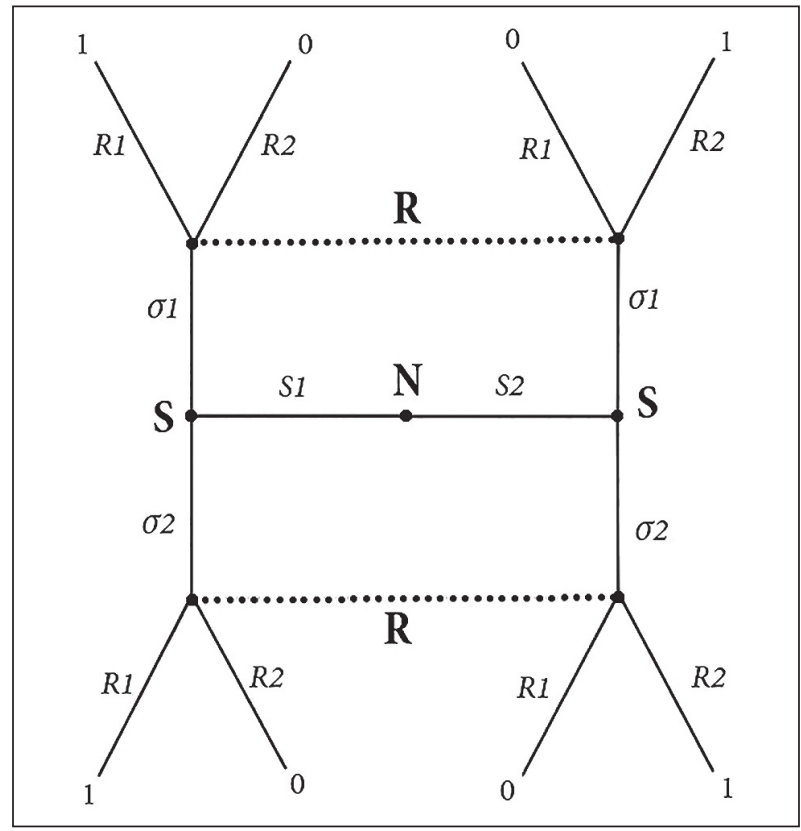

Fig. 1. A signaling system based on 2-states, 2-signals, 2-responses

The "nodes" of the tree represent the players (i.e., $\mathbf{N}$-nature, $\mathbf{S}$-sender, $\mathbf{R}$-receiver). The branches of the tree stand for events (i.e., $S 1, S 2$ - occurring states of affairs; $\sigma_{1}, \sigma_{2}$ - sending the signals by the sender; $R 1, R 2$ - taking actions by the receiver).

\section{Meaning in the signaling game framework}

The signaling game theory provides the code creation model for Eco's semiotic theory. The other side of code creation is the emergence of meaning from the equilibrium of the signaling game. This process takes place as a result of the underlying behavioral-signaling mechanisms, i.e. connections of signals with appropriate responses to them. The basis of this approach is the thesis according to which the meanings are evolutionary established conventions of response to a signal. But this raises a question of what is meant by meaning in the signaling game theory. A comprehensive and original answer to this question can be found in Skyrms' Signals. Evolution, Learning and Information (2010). However, in order 
to provide answers to the question: what is the meaning of signal (or an information content as Skyrms puts it), we must first find the answer to two related questions, namely: "What is the quantity of information in a signal?" and "How should it be measured?”.

\subsection{Quantity of information}

The concept of the quantity of information comes from the mathematical theory of information, which, as it is commonly known, does not deal with the informational content of a signal, since it understands the signaling process only in quantitative terms. According to mathematical theory of information, quantity is closely related to the value of probability that a given signal (stimulus) triggers a specific situation (reactions). As Skyrms puts it, the notion of information quantity can be easily applied to the signaling game model, since the occurrence of each of elements (events) within a game (i.e. state - signal - reaction) can be assigned to a certain probability (Skyrms, 2010: 34). Thanks to this feature, one can easily express the quantity of information carried by a signal as the ratio of the conditional probability of a particular state after sending a specific signal and the unconditional probability of this state (i.e. after sending the signal). This ratio gives an idea of how the probability of the state after sending the signal has changed with respect to the probability before the signal was sent. In formal terms, this may be expressed by the following equation (Skyrms, 2010):

$$
\frac{P(S \mid \sigma)}{P(S)}
$$

To illustrate this, let us assume that we have the simplest signaling game: 2-states, 2-signals, and 2-reactions, with an initial equal probability for each of them. This situation is a typical example of a state before reaching equilibrium (i.e. before the coding convention gets fixed). An increase in the level of the probability of a correct receiver's response to a specific signal corresponds to the achievement of equilibrium. In consequence, the signal becomes positively correlated with the state. A positive correlation increases also the probability of sending a specific signal $\left(\right.$ e.g., $\left.\sigma_{1}\right)$ any other time when that certain state occurs

\section{4 \&) COgnitiVE SCIENCE}


(e.g., $\left.S_{1}\right)$. Assume that the probability of this signal increases to a value of 0.9 . The probability of $\sigma_{2}$ signal in the event of the state $S_{1}$ is thus reduced to a value of 0.1 , since signals $\sigma_{1}$ and $\sigma_{2}$ are mutually exclusive events, and hence their total probability amounts to 1 . Of course, changing the probability of a signal doesn't affect the value of prior probability of the state $S_{1}$, and thus, it is still equal to 0.5 (i.e. $\left.P\left(S_{1}\right)=0.5\right)$. After reaching equilibrium, the conditional probability of signal $\sigma_{1}$ given state $S_{1}$ equals 0.9 (i.e. $P\left(\sigma_{1} \mid S_{1}\right)=0.9$ ). The cumulative probability of signal $\sigma_{1}$ is the sum of two products. The first of them is the unconditional probability of state $S_{1}$ multiplied by the conditional probability of signal $\sigma_{1}$ given state $S_{1}$. The second product is the unconditional probability of state $S_{2}$ times the conditional probability of signal $\sigma_{1}$ given state $S_{2}$. In formal terms, $P(\sigma)=\mathrm{P}\left(\sigma_{1} \cap S_{1}\right)+\mathrm{P}\left(\sigma_{1} \cap S_{2}\right)$ and it is equal to 0.5 . The overall probability distribution of our example is shown in fig. 2 in the form of a tree-diagram:

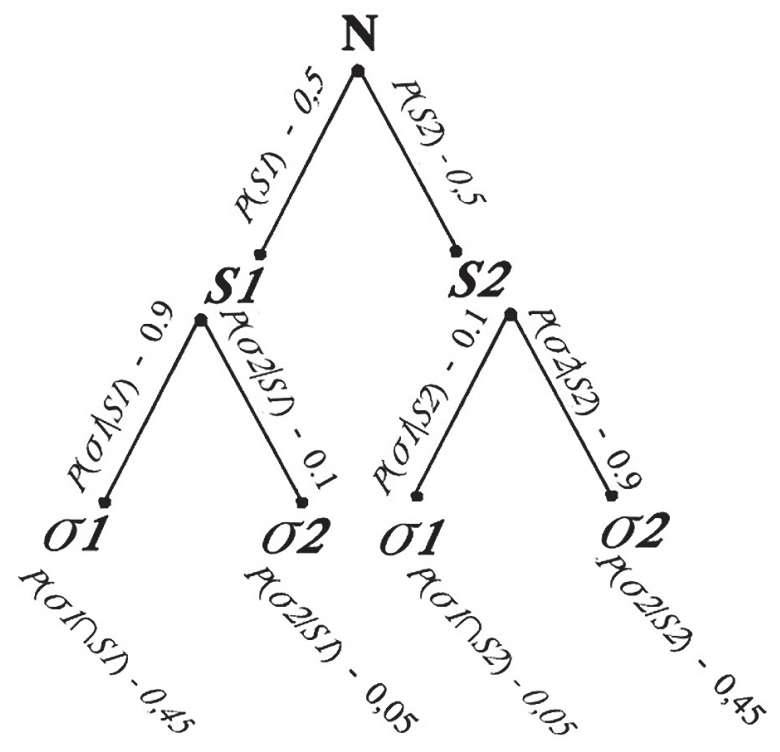

Fig. 2. Probability distribution of a signaling system of 2-states and 2-signals

By applying Bayes' theorem, we can easily calculate the value of the conditional probability of state $S_{1}$ after sending signal $\sigma_{1}$ : 


$$
\begin{gathered}
P\left(S_{1} \mid \sigma_{1}\right)=\frac{P\left(\sigma_{1} \mid S_{1}\right) \times P\left(S_{1}\right)}{P\left(\sigma_{1}\right)} \\
P\left(S_{1} \mid \sigma_{1}\right)=\frac{0,9 \times 0,5}{0,5} \\
P\left(S_{1} \mid \sigma_{1}\right)=0,9
\end{gathered}
$$

Therefore, the ratio of $P\left(S_{1} \mid \sigma_{1}\right)$ to $P\left(S_{1}\right)$ gives a value of 1.8 . This value expresses how many times the probability of state $S_{1}$ after sending signal $\sigma_{1}$ increases with respect to the prior probability of state $S_{1}$. It is the quantity of information carried by the signal. As Skyrms notices, we should take the logarithm of this ratio, since the quantity of information in the signal in present form does not allow us to express the situation when the signal does not convey any amount of information (e.g., if the sender always gives the same signal, regardless of the situation) (Skyrms, 2010: 36). In this case, the ratio of the conditional probability of the state after sending the signal to the prior probability of the state equals 1 , and not 0 , as we would intuitively expect. Thus, the final form of the information quantity formula is as follows (ibid.):

$$
\log _{2} \frac{P\left(S_{1} \mid \sigma_{1}\right)}{P\left(S_{1}\right)}
$$

Using the logarithm base 2 allows us to express the quantity of information in bits. This formula in its expanded form can be adapted to express information about the number of states, and to give a general measure of the information in the signal. Skyrms does this by using the Kullback-Leibler divergence formula, which expresses the average of the distribution of several probabilities (see: Kullback, Leibler, 1951; Skyrms, 2010):

$$
I\left(\sigma_{i} \mid S_{i}\right)=\sum_{i} P\left(S_{i} \mid \sigma_{i}\right) \log \left[\frac{P\left(S_{i} \mid \sigma_{i}\right)}{P\left(S_{i}\right)}\right]
$$

The above formula is simply a weighted average, in which the weights are the conditional probabilities of states given signals.

\section{6 \& COGNITIVE SCIENCE}


In addition to the quantity of information in the signal on a given state, we can still distinguish the amount of information in the signal on a given act. It is measured in the same way, as the amount of state, and expresses the ratio of the conditional probability of response after sending a signal to the probability of reaction before it is sent. The informational content in the signal carrying probability on the act takes the following form (Skyrms, 2010):

$$
I\left(\sigma_{i} \mid R_{i}\right)=\sum_{i} P\left(R_{i} \mid \sigma_{i}\right) \log \left[\frac{P\left(R \mid \sigma_{i}\right)}{P\left(R_{i}\right)}\right]
$$

Thus, the total amount of information in the signal consists of the two above values, i.e.

$$
I\left(\sigma_{i}\right)=I\left(\sigma_{i} \mid S_{i}\right)+I\left(\sigma_{i} \mid R_{i}\right)
$$

\subsection{Informational content}

Now let us turn to the issue of the information content of a signal. While the informational content in a signal is represented by a specified number (consisting of the sum of the amount of information about a state and about a reaction) expressed in bits, the information content of the signal is, according to Skyrms, represented by a vector whose components are the values of the informational content of states carried by the signal. The shape of the vector is related to a particular game. As Skyrms argues:

Informational content must be a vector $[\ldots]$ within a given signaling game. It is implicit that this vector applies to the states or acts of this game. For a different game, the content vector shows how the signal moves probabilities of different states, or different acts. Content depends on the context of the signaling interaction. It is a modeling decision as to which game is best used to analyze a real situation (Skyrms, 2010: 40).

The original proposal of expressing the informational content, as suggested by Skyrms, allows us to reconcile the information theory with the logical interpretation of propositional content, understood as a set of possible situations. The shape of the informational content vector is determined by the components formed from the values of informational quantity carried by the signal, and has the following form: 


$$
\left\lfloor\log \frac{P\left(S_{1} \mid \sigma_{1}\right)}{P\left(S_{1}\right)}, \log \frac{P\left(S_{2} \mid \sigma_{2}\right)}{P\left(S_{2}\right)}, \log \frac{P\left(S_{3} \mid \sigma_{3}\right)}{P\left(S_{3}\right)}, \ldots, \log \frac{P\left(S_{n} \mid \sigma_{n}\right)}{P\left(S_{n}\right)}\right\rangle
$$

A similar formula can be presented for the information content vector of actions carried by the signal.

$$
\left\lfloor\log \frac{P\left(R_{1} \mid \sigma_{1}\right)}{P\left(R_{1}\right)}, \log \frac{P\left(R_{2} \mid \sigma_{2}\right)}{P\left(R_{2}\right)}, \log \frac{P\left(R_{3} \mid \sigma_{3}\right)}{P\left(R_{3}\right)}, \ldots, \log \frac{P\left(R_{n} \mid \sigma_{n}\right)}{P\left(R_{n}\right)}\right\rangle
$$

To illustrate: suppose there are four states with an equal prior probability $P\left(S_{n}\right)=0.25$ for $n=\{1,2,3,4\}$, and that $\sigma_{2}$ signal is sent only when state $S_{2}$ occurs. This means that the value of $P\left(\sigma_{2} \mid S_{2}\right)$ is equal 1 , and the value of $P\left(\sigma_{2} \mid S_{1}\right)$, $P\left(\sigma_{2} \mid S_{3}\right)$, and $P\left(\sigma_{2} \mid S_{4}\right)$ equals 0 . Applying the Bayes' theorem, we get the value of the conditional probability of state $S_{2}$ after sending signal $\sigma_{2}$ equal to $1^{3}$. Thus, the probability of state $S_{2}$ after sending signal $\sigma_{2}$ increases four times with respect to the probability before the signal was sent:

$$
\frac{P\left(S_{2} \mid \sigma_{2}\right)}{P\left(S_{2}\right)}=\frac{1}{0,25}=4
$$

Since signal $\sigma_{2}$ is not sent in states $S_{1}, S_{3}$ and $S_{4}$ (i.e. the conditional probability of signal $\sigma_{2}$ for these states is 0$)$, it does not affect the probability of these states (i.e., $P\left(S_{1} \mid \sigma_{2}\right), P\left(S_{3} \mid \sigma_{2}\right)$ and $\left.P\left(S_{4} \mid \sigma_{2}\right)=0\right)$. Therefore, an increase in the level of probability for states $S_{1}, S_{3}$ and $S_{4}$ after sending the signal is 0 . If we take the logarithm of the values of all these probability ratios, we will obtain the informational content on all states in signal $\sigma_{2}$ :

$$
I\left(\sigma_{2} \mid S\right)=\langle-\infty ; 2 ;-\infty ;-\infty\rangle
$$

Component $-\infty$ informs us that the probability of states 1, 3 and 4 approach 0 . Value $-\infty$ is the result of the logarithm, and actually means that the signal does not carry any information about the state. But the question is: how to reconcile the above interpretation of the informational content with the logical in-

${ }_{3} P\left(S_{1} \mid \sigma_{1}\right)=\frac{P\left(\sigma_{1} \mid S_{1}\right) \times P\left(S_{1}\right)}{P\left(\sigma_{1}\right)}$.

\section{O) COGNITIVE SCIENCE}


terpretation of the meaning understood as a proposition? Presenting his model of informational content, Skyrms answers this question. The proposition is, in fact, a set of possible worlds or situations, so in his opinion, the informational content of a proposition can be expressed as a set of states. A state belonging to the set is true when its informational quantity carried by the signal varies from $-\infty$. In other words, the informational content or meaning of the signal is the set of possible situations or states which are true or false. The state is said to be true when the signal carries some informational quantity about it, and is recognized as false when it does not carry any quantity of information. As Skyrms points it out, a true state can be defined by listing all false states from the set of all states (2010: 41).

We believe that it is especially important that the signaling games model is compatible with the basic assumptions of Eco's theory of semiotics. According to our hypothesis, the signaling games theory can provide a model of code creation for Eco's semiotic theory. In other words, we suppose that denotation in semiotic theory - correlating a signal with a specific meaning - is preceded by a signaling system, which by correlating a signal with a proper reaction, makes it possible for a specific denotational coding convention to emerge. In our opinion, the core of this process is based on an evolutionary-fixed behavioral-signaling mechanism, i.e. connections of signals with appropriate responses to them.

However, the naturalistic-evolutionary theory of language is not sufficient as a descriptive model of the processes of the broadly-understood cultural activity evolution. The first problem is interpretation within the game theoretical framework of the culturally understood utility. From the point of view of biological interpretation, the numeric quantities, which play a role analogous to "utility" in traditional game theory, correspond to the Darwinian fitness of individuals. However, the Darwinian concept of "fitness" in the cultural evolutionary interpretation is inconsistent. Consequently, the concept of fitness as the notion of utility used in traditional game theory cannot be simply moved to the game theory of cultural evolution. One must develop an alternate theory of utility/fitness that is sufficient to define a utility measure adequate for application of evolutionary game theory to cultural evolution.

The second weakness of naturalistic theories involves high generative rigidity of new semantic content in the system. The content cannot be generated from within the semantic system, as it is possible in the case of the theory of semiotics, but requires reference to an external reality (reactions and states of affairs). 
The hybrid model proposed herein allows us to save the flexible potential of semiotic system, suitable for the explanation of cultural evolution on the one hand, and to root the semiotics in a natural order (by providing a model of emergence of evolutionary coding conventions) on the other. Such hybrid model requires, however, a formal tool to organize the semantic structure of the cultural system (in the signaling games framework, such tool is not needed, since the ordering of the meaning structure is carried by the values of informational quantity assigned to specific states). We will finish this paper by presenting a formal tool adequate for the task.

\section{Content implication}

The abovementioned tool is based on the propositional classical language extended by the binary intentional connective, called content implication, and represented by the sign of colon “:”. It was proposed by Piotr Łukowski $(1997,2011)$.

The Classical Logic with Content Implication (CLcont), based on language $L=\left(\right.$ For $\left._{U}, \neg, \wedge, \vee, \rightarrow, \leftrightarrow,:\right)$, is a propositional logic given by an axiom set for classical propositional logic and the following formulas:

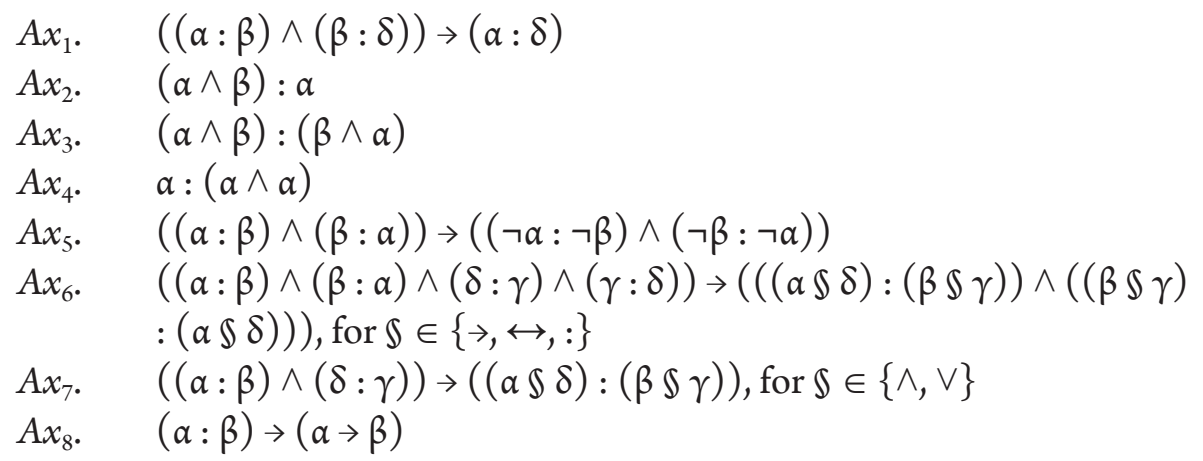

Modus Ponens (MP) $\{\rightarrow \beta, \alpha\} \mid-\beta$ is the only inference rule of $C L c o n t$. One of the most important CLcont-theses is $\alpha: \alpha$, a trivial formula easily inferred by $A x_{1}, A x_{2}$ and $A x_{4}$.

An adequate semantics for $C L$ cont is the class of all so-called CLcont-models, i.e., matrices $M=(A, D)$, such that $A=(A,-, \cap, \cup, \Rightarrow, \Leftrightarrow, \supset)$ is an algebra similar to $L_{U}, D$ is a nonempty subset of $A$ and for all $a, b \in A$,

\section{0 \&, COGNITIVE SCIENCE}


1. $a=a \cap a$

2. $a \cap b=b \cap a$

3. $-a \in D \quad$ iff $a \notin D$

4. $a \cap b \in D \quad$ iff $a D$ and $b \in D$

5. $a \cup b \in D \quad$ iff $a \in D$ or $b \in D$

6. $a \Rightarrow b \in D \quad$ iff $a \notin D$ or $b \in D$

7. $a \supset b \in D$ iff $a=b \cap c$, for some $c \in A$

Semantic inference is defined in a standard way:

$X \mid={ }_{U C l} \alpha$ iff $\quad$ for any CLcont-model $M=(A, D)$ and $v \in \operatorname{Hom}\left(L_{U}, A\right)$ $v(\alpha) \in D$, if for any $\beta \in X, v(\beta) \in D$.

According to the desired meaning of a new connective, $p: q$ is true if the content of sentence $q$ is included in the content of sentence $p$. Thus, sentence $p: q$ is true if and only if the content of $q$ is a part (not necessarily proper) of the content of $p$. In other words, $p: q$ is true, if sentence $p$ says what is said by $q$. Of course, $p$ can say something more than what is said by $q$. (Simultaneous truthfulness of $p: q$ and $q: p$ means that $p=q$ is true, and so $p$ says what is said by $q$ and $q$ says what is said by $p$ ).

The aim of construction of a new connective is simple: to express the fact that the content of one sentence is a part (not necessarily proper) of the content of another sentence. Therefore, the meaning of the new connective refers directly to the connective of conjunction. Truthfulness of sentence $p: q$ means that $p$ is a conjunction, in which $q$ is one of its conjuncts. In such a sense, the content of sentence $q$ is a part of the content of $p$. This feature makes content implication an excellent tool for organizing the semantic structure of a given system of propositions. At the same time, content implication turns out to be the perfect complement to the hybrid model postulated in this paper.

\section{Conclusion}

The objective of this paper was to compare structural semiotics with naturalistic semantics. The advantages and disadvantages of each were examined, and both approaches were shown to be complementary to one another, thereby providing a basis for further development of a hybrid system by combining the strengths of the two models. 


\section{References}

Bickerton, D. (2009). Adam's Tongue: How Humans Made Language, How Language Made Humans, New York: Hill \& Wang.

Bickerton, D., Szathmary, E. (ed.), (2009). Biological Foundations and Origin of Syntax, Cambridge, MA: MIT Press.

Brinck, I., Gärdenfors, P. (2003). Co-operation and communication in apes and humans. Mind \& Language 18 (5): 484-501.

Deacon, T.W. (1997). The Symbolic Species: Co-evolution of Language and the Brain. New York: W.W. Norton.

Eco, U. (1976). A Theory of Semiotics. Bloomington-London: Indiana University Press.

Gärdenfors, P. (2004). Cooperation and the evolution of symbolic communication. In: D.K. Ollera, U. Griebel, (eds.), Evolution of Communication Systems (pp. 237256). Cambridge: Cambridge University Press.

Kullback, S., Leibler, R.A. (1951). On information and sufficiency. Annals of Mathematical Statistics 22 (1): 79-86.

Lewis, D.K. (2002). Convention. A Philosopical Study. Oxford: Blackwell Chicago Press. Łukowski, P. (1997). An approach to the liar paradox. In: New Aspects in Non-Classical Logics and Their Kripke Semantics. RIMS: Kyoto University: 68-80.

Łukowski, P. (2011). Paradoxes. Dordrecht: Springer.

Ollera, D.K., Griebel, U. (ed.) (2004). Evolution of Communication Systems. Cambridge: Cambridge University Press.

Skyrms, B. (2010). Signals. Evolution, Learning and Information. New York: Oxford.

Shannon, C.E., Weaver, W. (1949). The Mathematical Theory of Communication. Urbana: University of Illinois Press. 